\title{
Preparación y densificación de monolitos ceramicos nanoestructurados de Y-TZP por Sol-gel
}

\author{
J. TARTAJ, J.F. FERNÁNDEZ, C. MOURE, E. LACHOWSKI ${ }^{1}$, P. DURÁN \\ Instituto de Cerámica y Vidrio (C.S.I.C.), Departamento de Electrocerámica, Arganda del Rey, 28500-Madrid. \\ ${ }^{1}$ University of Aberdeen, Department of Chemistry, Escocia, UK.
}

\begin{abstract}
Monolitos de geles de Y-TZP semillados han sido preparados por un método de sol-gel basado en la coprecipitación de precursores de $\mathrm{Zr}^{4+}$ y $\mathrm{Y}^{3+}$ en forma de hidróxidos y la estabilización de la suspensión en etanol puro con una turbina de disco. La adición de partículas nanométricas de Y-TZP cristalizadas a la solución precursora juega un papel fundamental durante la cristalización y densificación de los monolitos. Básicamente, se induce una nucleación preferente, que mejora la cinética de cristalización y reduce razonablemente la energía de activación. Como resultado del incremento de la frecuencia de nucleación para el proceso de cristalización, se obtiene una microestructura porosa, homogénea y bien desarrollada que facilita la sinterización y por tanto la densificación del material a temperaturas más bajas. De esta forma, monolitos cerámicos de Y-TZP altamente densificados han sido obtenidos a $1050^{\circ} \mathrm{C}$, con un tamaño de grano dentro de un rango nanométrico.
\end{abstract}

Palabras clave: Sol-gel, Semillado, Y-TZP, densificación, Nanoestructurado.

Preparation and Densification of Nanostructured Y-TZP Ceramic Monoliths by Sol-Gel.

Y-TZP seeded gel monoliths have been prepared by a sol-gel method based on the coprecipitation of $\mathrm{Zr}^{4+}$ and $\mathrm{Y}^{3+}$ precursors as hydroxides and the stabilisation of the suspension in pure ethanol with a disc turbine. The addition of solid Y-TZP nanometer-size seed particles into the Y-TZP precursor solution play a crucial role during cristallisation and densification of monoliths. Basically, induced a preferential nucleation, which enhance the crystallisation kinetics and the activation energy is reasonably lowered. As a result of the increased nucleation frequency for the crystallisation process a well-developed homogeneous porous microstructure is obtained facilitating the sintering and so the densification at lower temperatures. In this way, Y-TZP highly densificated ceramics monoliths have been obtained at $1050^{\circ} \mathrm{C}$, with a grain size within the nanometer range.

Keywords: Sol-Gel, Seeding, Y-TZP, Densification, Nanostructured.

\section{INTRODUCCION}

Uno de los conceptos más recientes en la búsqueda de nuevos materiales con nuevas características y propiedades y, por tanto, con nuevas aplicaciones tecnológicas son los materiales nanoestructurados. La importancia de éstos reside en que las propiedades de los materiales en una escala por debajo de 100 nm cambian drásticamente cuando se comparan con las obtenidas en los mismos materiales con tamaños de cristal superiores. El campo de las aplicaciones resulta enormemente amplio e interesante $(1,2)$. Se utilizan en catálisis heterogénea por la alta relación superficie/volumen de los materiales. Son materiales cerámicos dúctiles. El control del tamaño de poro permite la filtración selectiva e intercambio iónico. La estabilización de fases en sistemas polimórficos y de monodominios en materiales magnéticos (superparamagnetismo), además de la densificación de compactos nanocristalinos, son áreas de aplicación muy importantes. La disponibilidad de métodos económicos para producir grandes cantidades de estas sustancias y la alta energía almacenada en las nanoestructuras, constituyen el principal problema de estos materiales.

La obtención de materiales cerámicos con densidades próximas a la teórica y tamaño de partícula en el rango nanométrico es únicamente posible mediante la sinterización a temperaturas sensiblemente inferiores a las actualmente utilizadas, además del uso de polvos cerámicos altamente sinterizables y de óptimas condiciones de compactabilidad (3-5).

En general, los métodos de procesamiento químico como el sol-gel $(6,7)$ reúnen una serie de ventajas muy interesantes que pueden permitir la obtención de este tipo de materiales. Algunas de ellas son que la pureza de precursores iniciales conduce a una alta homogeneidad química en sistemas multicomponentes, distribución de tamaños de partículas muy estrecha, y sobre todo, que mediante la mezcla de precursores a un nivel atómico se rebajan las temperaturas de preparación. Sin embargo, en la obtención de geles poliméricos multicomponentes, la necesidad de disponer de los alcóxidos correspondientes y las distintas velocidades de hidrólisis son dificultades que hay que superar.

La circona policristalina tetragonal parcialmente estabilizada con óxido de itrio (Y-TZP) es uno de los materiales cerámicos más interesantes para aplicaciones estructurales. Incluso recientemente se han realizado ensayos satisfactorios de superplasticidad en estos materiales (8), en ellos se manifiesta la importancia de un tamaño de grano muy fino $(<1 \mu \mathrm{m})$.

En el presente trabajo se propone la obtención de monolitos de Y-TZP preparando de forma sencilla un gel de tipo coloidal que permite, tras su secado, la obtención de los mismos que son calcinados y sinterizados posteriormente. Por otra parte, se realiza el semillado de los geles, mediante la adición de 
nanopartículas de Y-TZP cristalizadas que favorecen la cinética de cristalización y reducen la energía de activación del proceso, permitiendo así un mejor control de la microestructura final del material $(9,10)$.

\section{PARTE EXPERIMENTAL}

Los precursores de $\mathrm{Y}$-TZP $\left(3 \mathrm{~mol} \% \mathrm{Y}_{2} \mathrm{O}_{3}\right)$ han sido preparados disolviendo cantidades adecuadas de $\mathrm{ZrOCl}_{2}$ y YCl $3.6 \mathrm{H}_{2} \mathrm{O}$ en una mezcla de agua/isopropanol, $1 / 1$ en volumen. Para el semillado, partículas cristalinas de Y-TZP ( $\left.{ }^{a} 6 \mathrm{~nm}\right)$, según se determinó por el análisis de difracción de rayos -X- utilizando la fórmula de Sherrer (11), fueron suspendidas en isopropanol puro y añadidas a la solución transparente de Y-TZP. Para asegurar una homogénea dispersión de las semillas en la solución precursora de Y-TZP, se sometió ésta a una intensa agitación que fue mantenida durante todo el proceso. Para precipitar los

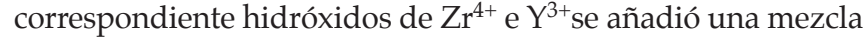
de isopropanol $+\mathrm{NH}_{4} \mathrm{OH}$ a la solución precursora que contiene las partículas (semillas) en suspensión. El precipitado gelatinoso obtenido, semillado con un $10 \%$ en peso, se lavó con butilamina en primer lugar para eliminar los cloruros y posteriormente con agua destilada y etanol puro, este último para eliminar la presencia de agua y así reducir las fuerzas de capilaridad que actúan sobre el gel durante el secado. Esta etapa juega un papel fundamental en la obtención de polvos secos con un muy bajo estado de aglomeración (12), y por tanto una buena capacidad de compactación. El coprecipitado así obtenido fue redispersado en etanol con una turbina de disco ultraturrax, que opera a 2000 r.p.m. proporcionando un fuerte flujo de líquido que conduce a una fuerte desaglomeracion del coprecipitado, permitiendo la estabilización de la suspensión por efecto mecánico. Además, al realizarse en etanol, grupos etóxido se unen a la superficie de las partículas previniendo la formación de enlaces entre las partículas (12). La suspensión coloidal así obtenida fue concentrada a $40-50^{\circ} \mathrm{C}$ manteniendo una ligera agitación, y fue entonces depositada en recipientes

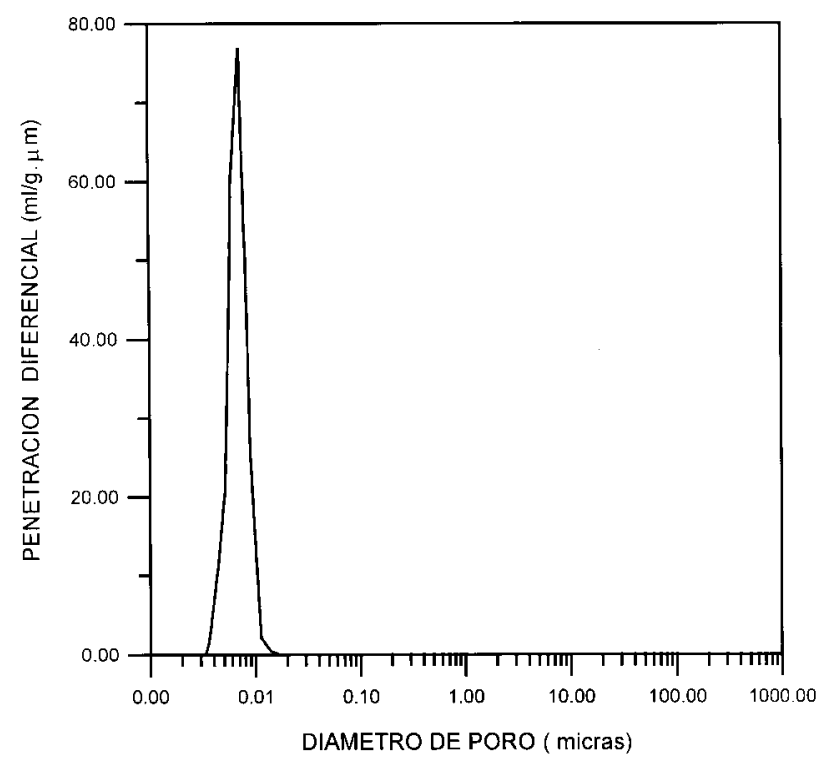

Fig. 1. Distribución del tamaño de poro en monolitos de Y-TZP semillados y calcinados a $500^{\circ} \mathrm{C}$ y $2 \mathrm{~h}$. de vidrio, dejándolo secar a $60^{\circ} \mathrm{C}$ durante 48 horas. Los monolitos $(1 \mathrm{~cm} \times 1 \mathrm{~cm} \times 0.2 \mathrm{~cm})$ así obtenidos fueron calcinados a $500^{\circ} \mathrm{C}$ durante 2 horas, con una velocidad de calentamiento de $1^{\circ} \mathrm{C} / \mathrm{min}$ y sinterizados a temperaturas de hasta $1400^{\circ} \mathrm{C}$ durante 5 horas a $2^{\circ} \mathrm{C} / \mathrm{min}$.

Las distribuciones del tamaño de poro de los monolitos calcinados se determinaron utilizando porosimetría de mercurio (Autopore II, 9215). Las densidades de los monolitos sinterizados se obtuvieron por el método de Arquímedes en agua destilada. La caracterización microestructural se realizó por microscopía electrónica de barrido y transmisión (TEM, Jeol 2000 FX).

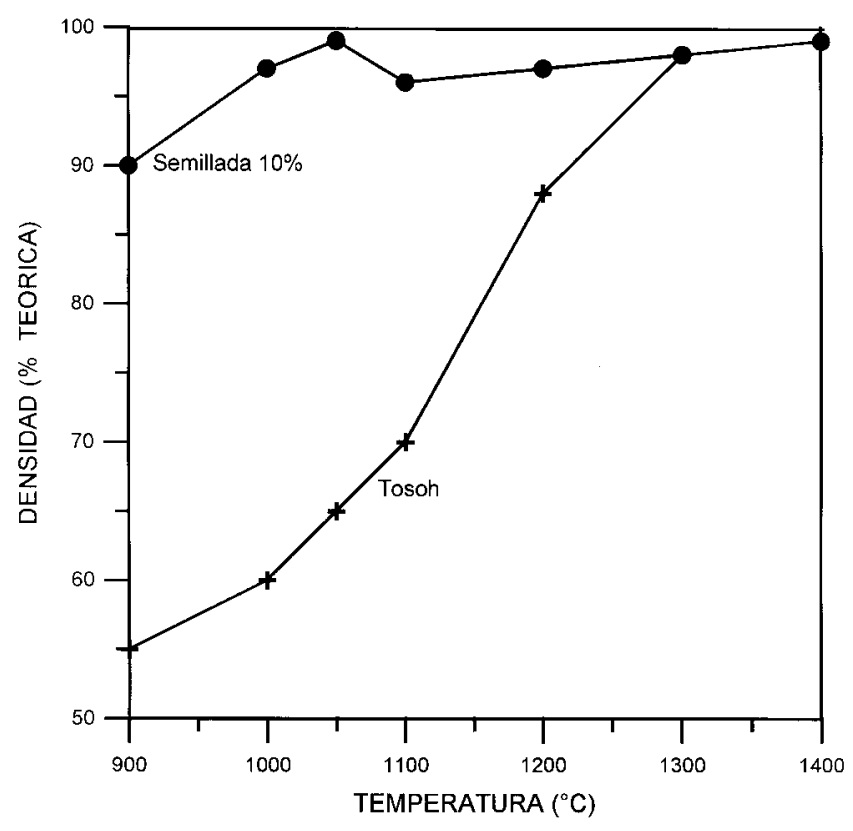

Fig. 2. Densidad frente a temperatura de sinterización de monolitos de Y-TZP semillados y comercial (Tosoh).

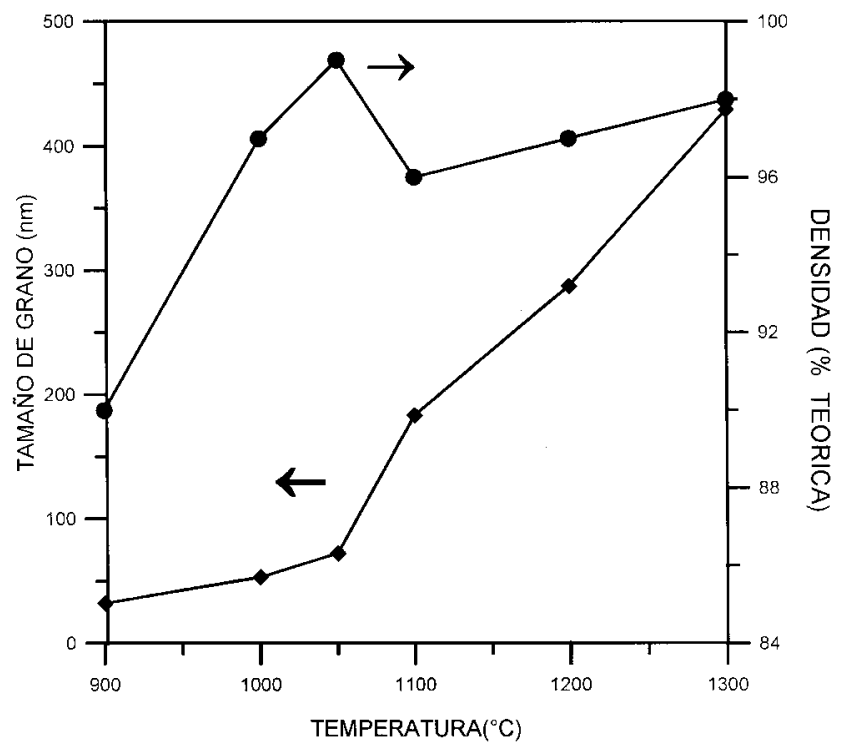

Fig. 3. Crecimiento de grano y densidad frente a temperatura de sinterización de monolitos de Y-TZP semillados. 


\section{RESULTADOS Y DISCUSION}

La Figura 1, muestra la distribución de tamaño de poro en monolitos calcinados a $500^{\circ} \mathrm{C}$. El tamaño medio de poro es de tan solo $7.5 \mathrm{~nm}$; además, la distribución del tamaño de poro es muy estrecha (20nm-3nm). Esta estrecha distribución parece ser consecuencia de la presencia de partículas de Y-TZP cristalizadas, las cuales reducen la energía de activación y por tanto favorecen la cinética de cristalización al incrementar la frecuencia de nucleación. De esta forma se obtiene una microestructura porosa muy homogénea que afecta de manera muy positiva al proceso de sinterización, siendo suficientes temperaturas más bajas para obtener monolitos altamente densificados. Resultados similares han sido referidos por McArdle y Messing (13) para la obtención de $\alpha-\mathrm{Al}_{2} \mathrm{O}_{3}$ a partir de geles de bohemita semillados con $\alpha-\mathrm{Fe}_{2} \mathrm{O}_{3}$.

La Figura 2, muestra la densidad en función de la temperatura de sinterización de los monolitos, comparativamente con una muestra comercial (Tosoh) ya estudiada (14). Las muestras se mantuvieron a cada temperatura durante $5 \mathrm{~h}$ con velocidades de calentamiento y enfriamiento de $2^{\circ} \mathrm{C} / \mathrm{min}$. En el caso de los monolitos semillados se observa una muy significativa mejora en la sinterización durante las primeras etapas del proceso $\left(<1000^{\circ} \mathrm{C}\right)$. Este comportamiento puede ser explicado como consecuencia de la cantidad de porosidad intercristalina presente, la cual es rápidamente eliminada y ya a $900^{\circ} \mathrm{C}$ la densidad es del $90 \%$. A $1050^{\circ} \mathrm{C}$ alcanzan ya un valor muy próximo a la densidad teórica $(\approx 99 \%)$, sin embargo entre $1100^{\circ} \mathrm{C}$ y $1200^{\circ} \mathrm{C}$ se observa un comportamiento anormal durante la sinterización, disminuyendo la densidad (96\%). Por encima de $1200^{\circ} \mathrm{C}$ y hasta $1400^{\circ} \mathrm{C}$ nuevamente la densidad alcanza valores superiores $(\approx 99 \%)$. Aunque varias causas pueden contribuir a este comportamiento de la densificación (15), el pequeño tamaño de partícula de los monolitos semillados y calcinados mejora la cinética de densificación en las primeras etapas de sinterización conduciendo a la obtención de monolitos densos a muy bajas temperaturas, (Figura 3). Además, el comportamiento de la densificación puede ser interpretado teniendo en

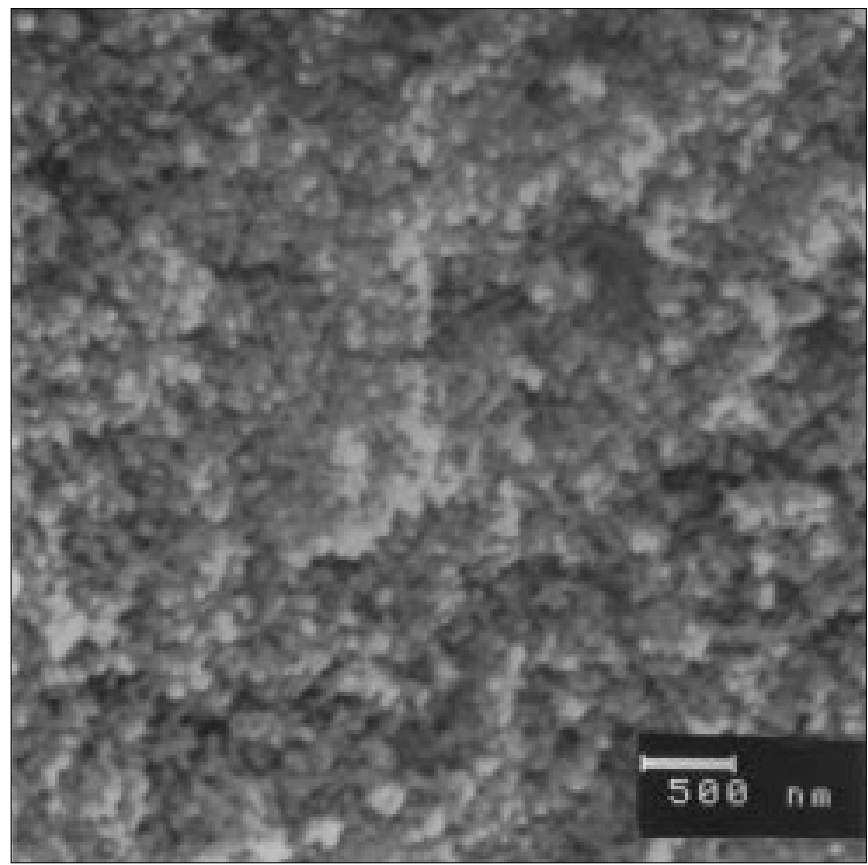

Fig. 4. Microestructura de fractura por MEB de monolitos a $900^{\circ} \mathrm{C}$. cuenta la relación crítica tamaño de poro y tamaño de grano postulado por Kingery et al. (15), mientras que hasta $1050^{\circ} \mathrm{C}$ la mayoría de los poros son muy pequeños respecto al tamaño de grano y por tanto fácilmente eliminables, ya que la relación tamaño de poro/tamaño de grano está por debajo de la crítica, lo que explica la rápida densificación. Por encima de $1050^{\circ} \mathrm{C}$ y hasta $1100^{\circ} \mathrm{C}$ tiene lugar una cierta coalescencia de poro; así, la relación tamaño de poro/tamaño de grano es superior al valor crítico con lo que la densidad disminuye, (Figura 3). A tratamientos superiores a $1100^{\circ} \mathrm{C}$ se produce un fuerte crecimiento de grano y nuevamente la relación tamaño de poro/tamaño de grano está por debajo del valor crítico, produciéndose una contracción con lo que la densidad vuelve a aumentar. En las muestras comerciales la densidad va aumentando de manera continua con la temperatura alcanzando su máxima densidad (> 99\%) a aproximadamente $1300^{\circ} \mathrm{C}\left(250^{\circ} \mathrm{C}\right.$ superior que las muestras semilladas). La presencia de aglomerados grandes con un relativamente pequeño tamaño de poro $(\approx 20 \mathrm{~nm})$ pero con una distribución uniforme desplaza la obtención de máximas densidades a temperaturas más altas (14).

La Figura 4, muestra la microestructura de fractura de los monolitos de Y-TZP semillados y sinterizados a $900^{\circ} \mathrm{C}$. La morfología y tamaño de poro son muy homogéneos y el tamaño de

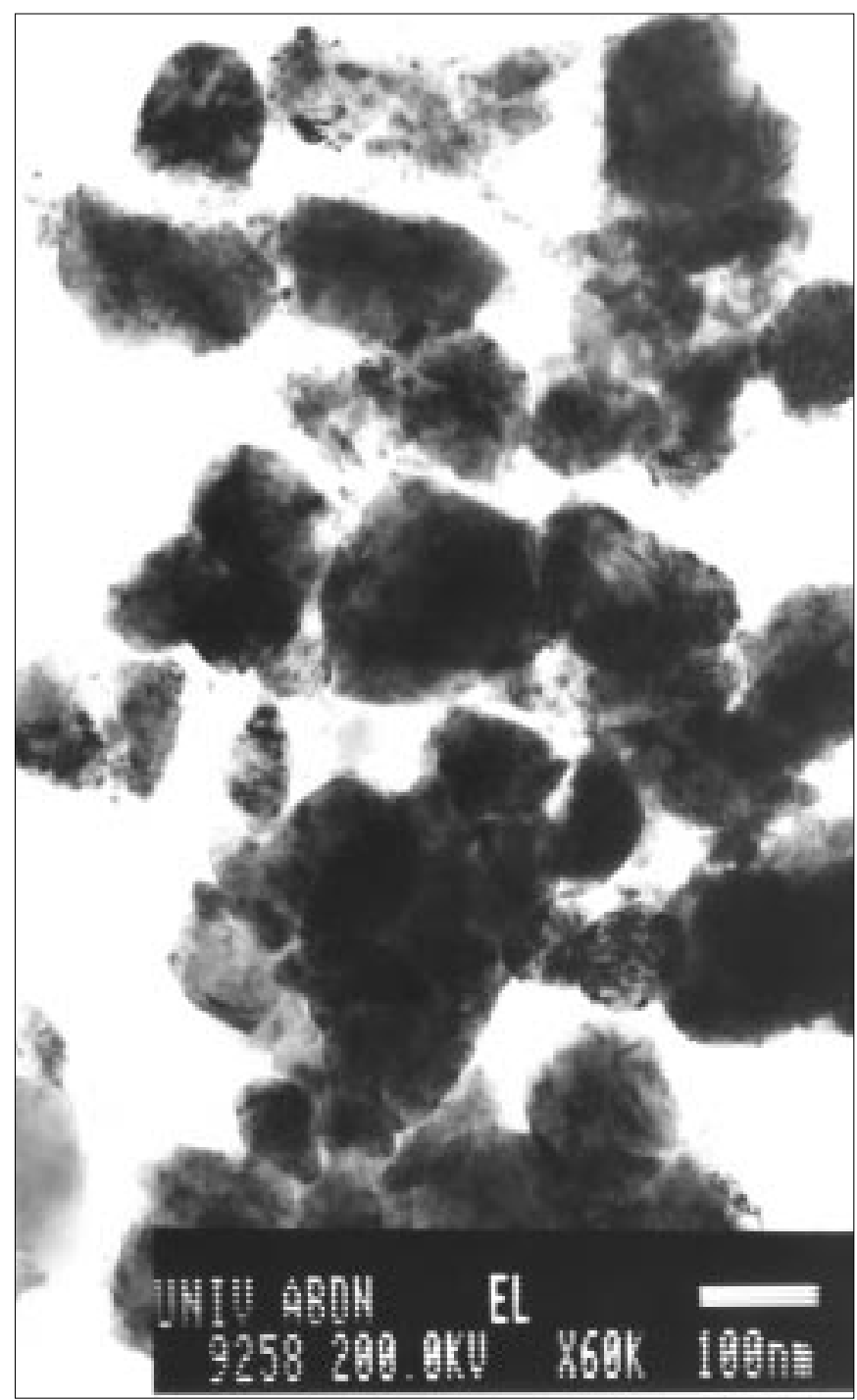

Fig. 5. Microestructura de superficie por MET de monolitos sinterizados a $1050^{\circ} \mathrm{C}$. 
grano se mantiene muy pequeño $(\leq 100 \mathrm{~nm})$. En el caso de la obtención de $\alpha-\mathrm{Al}_{2} \mathrm{O}_{3}$ a partir de bohemita y semillada con $\alpha-\mathrm{Fe}_{2} \mathrm{O}_{3}(16,17)$ se explica el efecto de la adición de las semillas. Estas incrementan la frecuencia de nucleación y por tanto, el volumen de material transformado por cada punto de nucleación se reduce; así, el área o fracción de borde de grano se incrementa mejorando de esta forma el proceso de densificación. Por el contrario, si la frecuencia de nucleación es baja, y por tanto, el volumen de material transformado por punto de nucleación aumenta; al producirse una considerable contracción durante la transformación a $\alpha-\mathrm{Al}_{2} \mathrm{O}_{3}$, o como en nuestro caso durante el proceso de cristalización; aparece una alta fracción de porosidad remanente alrededor de estas amplias áreas de transformación que dificultan notablemente la densificación del material. Por tanto, la presencia de estas partículas sobre las que se induce el proceso de cristalización, al ser puntos de nucleación energéticamente favorables reduce de manera significativa la porosidad, facilitando la eliminación de ésta y permitiendo la densificación de estos monolitos de Y-TZP a temperaturas realmente bajas que permiten controlar de manera efectiva el tamaño de grano y obtener así, materiales ya sinterizados y altamente densificados en un rango prácticamente nanométrico, como muestra la micrografía de la Figura 5 obtenida por microscopía de transmisión electrónica de muestras sinterizadas a $1050^{\circ} \mathrm{C}$. Estos materiales, por sus características, podrían presentar un comportamiento superplástico aplicable en áreas de alta tecnología como el conformado a alta temperatura.

\section{CONCLUSIONES}

Monolitos de Y-TZP han sido preparados mediante un sencillo método basado en la coprecipitación de precursores de $\mathrm{Zr}^{4+} \mathrm{y} \mathrm{Y}^{3+}$ en forma de hidróxidos y, la posterior estabilización de la suspensión obtenida tras la coprecipitación por medios mecánicos en un medio alcohólico adecuado. La adición de partículas de Y-TZP al gel conduce a la formación de monolitos, que una vez calcinados, presentan una distribución de tamaño de poro muy estrecha $(7.5 \mathrm{~nm})$. El tratamiento térmico de éstos a temperaturas relativamente bajas conduce a la obtención de materiales de Y-TZP nanoestructurados y de densidades del 99\%.

\section{AGRADECIMIENTOS}

J. Tartaj agradece la Beca de Intercambio recibida, Royal Society-CSIC, para la realización de parte de este trabajo en la Universidad de Aberdeen, Escocia.

\section{BIBLIOGRAFIA}

1. Y. M. Chiang. "Introduction and Overview: Physical Properties of Nanostructured Materials". J. Electroceram. 1 205-209 (1997).

2. M. Sternitzke. “Review: Structural Ceramic Nanocomposite". J. Europ. Ceram. Soc. 17 1061-1082 (1997).

3. E. Tani, M. Yoshimura, and S. Somiya. "Formation of Ultrafine Tetragonal Zirconia Powders under Hydrothermal Conditions". J. Am. Ceram. Soc. 66 (1) 11-14 (1983).

4. M. M. R. Boutz, R. J. M. Olde Scholtenhuis, A. J. A. Winnubst and A. J. Burggraaf. "A Hydrothermal Route for Production of Dense Nanostructured Y-TZP". Mater. Res. Bull. 29 31-40 (1994).

5. G. Skandan, H. Hahn, M. Rooddy and W. R. Cannon. “Ultrafine Grained Dense Monoclinic and Tetragonal Zirconia". J. Am. Ceram. Soc. 77 1706-1710 (1994).

6. J. D. Mackenzie. “Applications of the Sol-Gel Process". J. Non Crys. Solids. 100 162-168 (1988).

7. D. P. Partlow and B. E. Yoldas. “Colloidal versus Polymer Gels and Monolithic Transformation in Glass-Forming Systems". J. Non Crys. Solids. 46 153-161 (1981).

8. M. M. R. Boutz, A. J. A. Winnubst, A. J. Burggraaf, M. Nauer and C. Carry. "Low Temperature Superplastic Flow of Y-TZP". J. Europ. Ceram. Soc. 13 103-111 (1994).

9. J. Tartaj, J. F. Fernández, C. Moure and P. Durán. “Influence of Seeding on the Crystallisation Kinetics of Air-Calcined Y-TZP Gel-Derived Precursors". Mat. Res. Bull. 32 1525-1533 (1997).

10. J. Tartaj, J. F. Fernández, C. Moure and P. Durán. "Effects of Seeding on the Crystallisation Kinetics of Air-Calcined Yttria-Doped Hydrous Zirconia". J. Europ. Ceram. Soc. 18 229-235 (1998).

11. K. P. Klug and L. E. Alexander. "Crystallite Size and Lattice Strains from Line Broadening", pp. 618-708 en X-ray Diffraction Procedures, Chap. 9. Ed. John Wiley \& Sons, New York, (EE.UU.) 1974.

12. P. D. L. Mercera, J. G. Van Ommen, E. B. M. Dresbrug, A. J. Burggraaf and J. R. H. Ross. "Influence of Ethanol Washing of the Hydrous Precursor on the Textural and Structural Properties of Zirconia". J. Mater. Sci. 27 4890-4898 (1992).

13. J. L. McArdle and G.L. Messing. "Transformation, Microstructure Development, and Densification in $\alpha$ - $\mathrm{Fe}_{2} \mathrm{O}_{3}$-Sedeed Boehmite-Derived Alumina". J. Am. Ceram. Soc. 76[1] 214-222 (1993).

14. P. Durán, J. Tartaj, J.F. Fernández, M. Villegas and C. Moure. “Crystallisation and Sintering Behaviour of Nanocrystalline Y-TZP Powders Obtained by Seeding-Assisted Chemical Coprecipitation". Ceramics Intern., 25 (1999) 125135.

15. W. D. Kingery, H. K. Bowen and D. R. Uhlmann. p. 486 en Introduction to Ceramics, $2^{\text {nd }}$ edition. Ed. John Wiley \& Sons, New York, (EE.UU) 1976.

16. J. L. McArdle and G. L. Messing. "Transformation and Microstructure Control in Boehmite-Derived Alumina by Ferric Oxide Seeding". Adv. Ceram. Mater. 3[4] 387-392 (1988).

17 J. Tartaj. "Proceso de Nucleación Heterogénea de la Fase a- $\mathrm{Al}_{2} \mathrm{O}_{3}$ sobre Partículas Cristalográficamente Adecuadas $\left(\alpha-\mathrm{Fe}_{2} \mathrm{O}_{3}\right)^{\prime}$. Bol. Soc. Esp. Ceram. Vid. 35[6] 429-432 (1996). 